\title{
Beten mit Luhmann
}

\section{Zu einer systemtheoretischen Deutung des christlichen Gebets}

Stefan Berg

\section{Hinführung}

Wollte man unter den grossen Denkern des 20. Jahrhunderts denjenigen mit dem sperrigsten Ansatz küren, so ist Niklas Luhmann ein aussichtsreicher Kandidat. Seine Systemtheorie gleicht einem sehr umfangreichen und in seiner Knüpfung zugleich überaus engmaschigen Netz. Es scheint mit dem Anspruch geknüpft, dass sich darin so ziemlich alle entscheidenden Aspekte menschlichen Lebens verfangen, und es kommt einem mitunter so vor, als würde es von Luhmann und seinem Gefolge mitunter in der Überzeugung ausgeworfen, dass alle anderen Fischer nicht nur mit kleineren und weniger tauglichen Netzen operieren, sondern sich auch die grössten unter ihnen letztlich als hilflos zappelnder Fang auf dem grossen Bielefelder Trawler wiederfinden werden.

Für denjenigen, der Luhmann weder einfach ignorieren will, noch sich mit ihm einen Kampf der Kapitäne liefern, für den also, der seine Theorie schlicht redlich und eigenständig zu rezipieren gedenkt, schafft diese Gemengelage Probleme. Selbst wenn man sich den kritischen Blick auf Luhmanns Fangmethode bewahrt, so ist es alles andere als einfach, in seinem Netz eine Schlaufe zu finden, an die man selbst anknüpfen kann, ohne gleich das ganze Maschenwerk um den Hals gewickelt zu bekommen. Es wäre ja beispielsweise methodisch fragwürdig, einen einzelnen Aspekt etwa der Religion der Gesellschaft herauszugreifen, ohne auch die Beobachtertheorie, den Autopoiesisgedanken oder die Reentryfigur zu übernehmen. Bei Luhmann hängt scheinbar alles mit allem zusammen, und so endet man schnell als willfähriger Leichtmatrose unter seinem Kommando.

Ein Aspekt dieses Problems zeigt sich in der theologischen Rezeption Luhmanns. Man liest eine Schrift wie die Religion der Gesellschaft ja durchaus mit Gewinn und fühlt sich als Christenmensch darin auch in produktiver Art und Weise beschrieben. Aber man fühlt sich zugleich nicht ganz richtig verstanden, und hat das Bedürfnis, eigene 
Sichtweisen kritisierend oder differenzierend eintragen zu können.Und genau dazu bietet der engmaschig-geknüpfte Ansatz Luhmanns offenbar wenige Spielräume.

Vor diesem Hintergrund stelle ich mit dem Gebet ein klassisches materialdogmatisches Thema ins Zentrum meiner folgenden Überlegungen. Ich werde anhand seiner erproben, ob und wie sich ein theologischer Umgang mit Luhmann finden lässt. Das Gebet eignet sich für eine solche Erkundung in vorzüglicher Weise, weil sich an ihm in einer Deutlichkeit wie vielleicht an keinem anderen Phänomen christlichen Lebens theologische Grundfragen auskristallisieren - insbesondere: wie Gott, Mensch und deren Beziehung zu einander zu denken sind und wie sich dies im Leben im christlichen Glauben konkret realisiert.

\section{Luhmanns Frage: Kann die Systemtheorie Kommunikation mit Gott denken?}

Luhmanns Äusserungen zum Gebet sind spärlich. Etwa in Funktion der Religion, Die Religion der Gesellschaft und auch im gemeinsam mit Peter Fuchs verfassten Reden und Schweigen wird das Gebet nur marginal thematisch. Bedenkt man, welch wichtige Rolle das Gebet in der alltäglichen Lebenspraxis von Christenmenschen spielt, und stellt man in Rechnung, dass Niklas Luhmann von Haus aus Soziologe ist, so darf dieser schlichte, quantitative Befund doch ein wenig überraschen.

Neben den genannten Schriften gibt es immerhin einen kleinen Text Luhmanns, der sich etwas eingehender mit dem Gebet beschäftigt und die Karten offen auf den Tisch legt. Er trägt den Titel Läßt unsere Gesellschaft Kommunikation mit Gott zu? und setzt sprechenderweise mit einem "Bekenntnis zum Nichtbekenntnis ${ }^{1}$ ein, um schon bald unmissverständlich deutlich zu machen, warum das Gebet für den Autor eben tatsächlich ein nebensächliches Thema ist: "Es gibt $[\ldots]$ keine Kommunikation mit Gott ${ }^{2}$. - Wie kommt er zu diesem apodiktischen Urteil?

Letztlich erleidet die Möglichkeit einer positiven Würdigung des Gebets an Luhmanns Gottesbegriff Schiffbruch. Um dies nachzuvollziehen, muss man zunächst verstehen, dass als Zentralelement

1 Niklas Luhmann, Läßt unsere Gesellschaft Kommunikation mit Gott zu?, in: Soziologische Aufklärung 4. Beiträge zur funktionalen Differenzierung der Gesellschaft, Opladen 1987, 227-235, hier 227.

2 Luhmann, Kommunikation mit Gott, 229. 
seiner Religionstheorie nicht Gott, sondern die Unterscheidung von Immanenz und Transzendenz fungiert. Auf diesen Code lassen sich alle Kommunikationsvollzüge beziehen, in denen sich das Religionssystem autopoietisch konstituiert und auf diese Weise innerhalb der Gesellschaft die Funktion übernimmt, unbestimmbare in bestimmbare Komplexität zu transformieren.

Um das Auseinanderfallen des binären Codes zu verhindern, von dem jedes Teilsystem der Gesellschaft als eine Art Rückgrat aufrecht gehalten wird, muss es in jedem dieser Teilsysteme eine Instanz geben, die es erlaubt, »beide Seiten der Unterscheidung als Einheit $\mathrm{zu}$ thematisieren « ${ }^{3}$. Diese Funktion übernehmen die sogenannten Kontingenzformeln, die deshalb ihren Namen tragen, weil sie die genannte einheitsstiftende Funktion erfüllen, indem sie die Kontingenz des Codes im jeweiligen System absorbieren. In monotheistischen Spielarten des Religionssystems fungiert Gott als eine solche Kontingenzformel. Das heisst: In der Kommunikation des Religionssystems unterdrückt die Thematisierung Gottes nicht nur die Fragen, warum man überhaupt zwischen Immanenz und Transzendenz unterscheide und ob man nicht auch anders unterscheiden könnte, sondern sie erlaubt es auch, Selektionskriterien zu generieren, wie ein richtiger oder falscher Umgang mit der Unterscheidung von Immanenz und Transzendenz aussehe. ${ }^{4}$

Nun kennt der christliche Glaube nicht bloss die Möglichkeit, über Gott zu kommunizieren, sondern zieht auch diejenige in Betracht, dies mit Gott zu tun. Hier jedoch wird es für Luhmann hochproblematisch. Ist Kommunikation mit Gott im Horizont der Systemtheorie denkbar?

Ganz auf der Linie seines eigenen Ansatzes formuliert Luhmann diese Frage nicht dergestalt, ob ein Mensch, der im Glauben lebt, mit Gott kommunizieren könne. "Der Mensch kann" in seiner Sicht nämlich gar »nicht kommunizieren; nur die Kommunikation kann kommunizieren " ${ }^{5}$. Das heißt, nicht der Mensch bedient sich der Kommunikation, sondern die Kommunikation bedient sich des Menschen. Die Instanz >Mensch im Glauben` wäre für ihn daher wohl reine Struktur der Autopoiesis des Religionssystems`, nicht aber eine irgendwie spontane bzw. souveräne Entität, von der etwas

3 Bernd Oberdorfer, Kontingenzformel `Gottr. Der christliche Gottesgedanke unter systemtheoretischer Beobachtung - trinitätstheologisch beobachtet, in: Luhmann und die Theologie, hg. v. Günter Thomas/Andreas Schüle, Darmstadt 2006, 107-115, hier 108.

${ }^{4}$ Vgl. Niklas Luhmann, Die Religion der Gesellschaft, hg. v. André Kieserling, Frankfurt a.M. 2002, 167f.

5 Niklas Luhmann, Die Wissenschaft der Gesellschaft, Frankfurt a.M. 1992, 31. 
Konstitutives abhängen könnte. ${ }^{6}$ Und so fragt Luhmann mit dem Titel des hier referierten Textes: Läßt unsere Gesellschaft Kommunikation mit Gott zu? - eine etwas unglücklich holpernde Formulierung, weil es im Aufsatz ja nicht um die Frage einer Zulassung der Kommunikation mit Gott geht, sondern eher um diejenige nach der systemtheoretischen Legitimierbarkeit der Vorstellung einer Kommunikation mit Gott.

\section{Luhmanns Antwort: Kommunikation mit Gott ist systemtheoretisch nicht denkbar}

Demnach lässt sich auch die Beantwortung der Frage, ob Kommunikation mit Gott denkbar sei, nicht von den Vollzügen lösen, die Gesellschaft konstituieren. Als basale Prämisse stellt Luhmann zunächst einmal klar, dass diese nur in und als Kommunikation existiert:

"Wo immer Kommunikation versucht wird, bildet sich daher Gesellschaft im Sinne einer Ordnung von Nichtbeliebigkeiten der Kommunikation. Alle Kommunikation ist ein gesellschaftsinterner Vorgang, jede Kommunikation vollzieht Gesellschaft, und wo Kommunikation aufhört, da hört auch Gesellschaft auf. Es gibt in der Umwelt der Gesellschaft keine Kommunikation, gäbe es irgendwo anders Kommunikation, würde deren Wahrnehmung zugleich Wahrnehmung der Ausdehnung des Gesellschaftssystems sein. Aus dem gleichen Grunde gibt es auch keine Kommunikation zwischen dem Gesellschaftssystem und seiner Umwelt [...]. Die Gesellschaft kann nicht mit ihrer Umwelt, sie kann nur über ihre Umwelt kommunizieren. ${ }^{7}$

Unmittelbar auf die damit vorgenommene Identifikation der Grenze der Kommunikation mit der Grenze der Gesellschaft folgt sofort und geradezu unvermittelt die Beantwortung der entscheidenden Frage:

"Es gibt also keine Kommunikation mit Gott. Weder kann man sich Gott vorstellen als jemanden, der zu Noah, Abraham, Jakob oder anderen spricht; noch kann man ihn denken als jemanden, der angerufen werden kann (wenn man nur seinen Namen weiß). Und in letzter Konsequenz heißt dies schließlich, daß weder Offenbarung noch Gebet als Kommunikation zu denken sind.

6 Ich formuliere hier in Anlehnung an Luhmann, Wissenschaft, 33.

7 Luhmann, Kommunikation mit Gott, 228. 
Alle diese Vorstellungen führen letztlich zu einer Eingemeindung Gottes in die Gesellschaft. Sie mögen Trost und Erbauung bieten und ideenpolitisch schwer zu entbehren sein. Sie sind jedoch eine Dauerquelle der Reproduktion anthropomorpher Religionsvorstellungen, die dann theologisch wieder diskreditiert oder mit hinreichender Ambivalenz versehen werden müssen. Und nicht zuletzt blockieren sie ein auf Theorie und begriffliche Klarheit gegründetes Gespräch zwischen Theologie und Soziologie. $" 8$

Dieser auf den ersten Blick etwas aus der Hüfte geschlossene Schluss lässt sich besser nachvollziehen, wenn man die binäre Anlage der Gesamtkonstellation hervorhebt. Für die eine Seite ist zu bemerken, dass Gott, also die als Einheit der Unterscheidung von Immanenz und Transzendenz fungierende Kontingenzformel, von der Religion selbst in der Transzendenz angesiedelt wird. ${ }^{9}$ Von hier aus kann sie Kontingenz besonders glaubhaft absorbieren, weil sie dort aller Kontingenz des Immanenten enthoben ist. Gott und Transzendenz bilden also den einen Zusammenhang. Auf der anderen Seite installiert Luhmann einen antagonistischen Komplex aus Immanenz, Gesellschaft und Kommunikation. Dass Gesellschaft und Kommunikation zusammenfallen, ist - wie im Zitat deutlich - eine Kernprämisse von Luhmanns Ansatz, und dass die Gesellschaft furr ihn als Soziologen eine immanente und keine transzendente Grösse ist, kann kaum überraschen - wobei man hier als Theologe anmerken muss, dass es etwa mit Augustins De civitate Dei durchaus theologische Ansatzpunkte für alternative Modelle gibt.

Unter den so skizzierten Umständen impliziert es nun tatsächlich einen Selbstwiderspruch, würde man vertreten, dass immanente Kommunikation dem transzendenten Außerhalb der Kommunikation kommunikativ begegnen könne. Denn wohin die Kommunikation vordränge, da wäre Kommunikation, d.h. Immanenz, und nicht ihr transzendentes Außerhalb. Oder anders gewendet: Das transzendente Außerhalb der Kommunikation wiche selbstverständlich vor jedem Versuch der Ausweitung der Kommunikation zurück und könnte von ihm nie erreicht werden. Dass Kommunikation aus der Immanenz heraus mit Gott als dem transzendenten Außerhalb der Kommunikation möglich sei, wäre also in der Tat ein Ungedanke: die Vorstellung einer >Eingemeindung Gottes in die Gesellschaft‘.

\footnotetext{
8 Ebd., 229.

9 Vgl. Niklas Luhmann, Die Unterscheidung Gottes, in: Soziologische Aufklärung 4. Beiträge zur funktionalen Differenzierung der Gesellschaft, Opladen 1987, 236-253.
} 
In die Argumentation ist ein weiterer Begründungsaspekt eingeflochten. Die Annahme, dass mit Gott kommuniziert werden könnte, befördert für Luhmann personal-anthropomorphe Missverständnisse. Wie man in der Religion der Gesellschaft genauer nachlesen kann, ${ }^{10}$ repräsentieren derartige Vorstellungen nach Ansicht Luhmanns eine gewissermassen veraltete Religionsauffassung, die diverse Probleme nach sich zieht, ${ }^{11}$ insbesondere dasjenige, dass die doch eigentlich kontingenzabsorbierende Kontingenzformel mit Kontingenz aufgeladen und zu jemandem wird, mit dem man diskutieren und streiten kann, wie es zum Beispiel Hiob versucht.

Im hier referierten Kontext deutet Luhmann diesen Hintergrund nur an. Entsprechendes spielt aber auch dann eine gewisse Rolle, wenn Luhmann durchblicken lässt, dass er durch interdisziplinäre Gespräche zwischen Theologie und Soziologie gern insbesondere solche "Formen von Religion" gefördert sähe, »die den typischen Strukturen der modernen Gesellschaft mehr entsprechen als andere ${ }^{12}$ - und das heißt nichts anderes als: deren Selbstbeschreibung mit Luhmanns Theorie vollkommen zur Deckung käme und alle personal-anthropomorphen Aspekte konsequent negierten. An Luhmanns Wesen soll die Theologie genesen.

Luhmann spielt in seiner Beschäftigung mit Religion offensichtlich nicht bloss die Rolle des deskriptiv distanzierten Soziologen. Er verfolgt eine normative Religionstheorie, die von seinen anti-metaphysischen bzw. anti-subjektivistischen Prämissen getragen wird, wie er sie zum Beispiel in der Wissenschaft der Gesellschaft entfaltet. Es ist so gesehen wohl kein Zufall, dass Luhmanns Aufsatzband, aus dem unser Text zur Kommunikation mit Gott stammt, den Titel Soziologische Aufklärung trägt. Argumentationsstrategisch betrachtet erinnert nämlich einiges an Kants Religionsphilosophie. Kant und Luhmann treffen sich nicht nur material in der Ablehnung des Gebets - denn man kann ebenso wenig mit einer regulativen Idee wie mit einer Kontingenzformel kommunizieren -, sondern auch darin, dass sie die Entwicklung hin zu einer transzendental bzw. systemtheoretisch aufgeklärten Religion unterstützen und eben zum Beispiel anthropomorph-personale Aspekte im Gottesbild bekämpfen. ${ }^{13}$

Der normative Anspruch wird schliesslich mit einer religionsgeschichtlichen Skizze unterfüttert, die zu zeigen beansprucht, »daß im

\footnotetext{
${ }^{10}$ Vgl. Luhmann, Religion der Gesellschaft, 150-154.

${ }_{11}$ Vgl. Oberdorfer, Kontingenzformel Gott, 108-110.

${ }^{12}$ Luhmann, Kommunikation mit Gott, 229.

${ }^{13}$ Vgl. Immanuel Kant, Die Religion innerhalb der Grenzen der bloßen Vernunft, A 284-289.
} 
Laufe der gesellschaftlichen Evolution die Vorstellung einer Kommunikation mit Gott immer schwieriger wird ${ }^{14}$, will sagen: immer unselbstverständlichere Interpretationsleistungen vornimmt, um am Kommunikationsbegriff festhalten zu können.

\section{Ausnahmezustandsrhetorik und Kontingenz}

Bis hierhin erscheinen Luhmanns Überlegungen kaum des Referats wert, sind sie doch im Wesentlichen systemtheoretische Reformulierungen altbekannter religionsphilosophischer Bedenken hinsichtlich derVermittelbarkeit von Immanenz und Transzendenz.

Mit der Diskussion der Frage, wie die Theologie angesichts der skizzierten Vorbehalte am Kommunikationsbegriff festhalten könne, gerät Luhmann aber auf eine andere Ebene. Dies geschieht zunächst in Gestalt einer Provokation: "Aber wozu [sc. am Kommunikationsbegriff festhalten, d.V.]? - wenn man ihn [sc. Gott] weder über etwas informieren kann, was er noch nicht weiß, noch erwarten kann, daß die Kommunikation ihn zu etwas motivieren könnte, was er andernfalls nicht tun würde. « ${ }^{15}$ Und er fährt im nächsten Absatz fort:

»Ich wähle für diese Fragen bewußt eine Form, die unterhalb der Ebene bleibt, auf der Theologen diese Frage behandeln würden. Es kommt mir aber darauf an, die Theologie zu einer Stellungnahme zu bewegen. Es genügt mir nicht, wenn ich die Antwort erhalte, Kommunikation mit Gott sei etwas ganz anderes als Kommunikation unter Menschen. Wenn so, dann sollte man einen anderen Ausdruck dafür wählen. Denn wenn man Kommunikation sagt und zugleich sagt, daß man nicht meint, was man sagt, handelt man im strengen Sinne paradox; denn dann kann man wissen, da[ $[\beta]$ der andere nicht wissen kann, was man meint, daß man nicht meint, was man sagt. $\|^{16}$

Mit diesem Vorwurf dürfen wir es uns als Theologinnen und Theologen nicht zu einfach machen. Die dialektisch-theologische Rede von Gott als dem Ganz-Anderen hat ja eine gewisse façon de parler hoffähig gemacht, die einen Ausnahmezustand rhetorisch auf Dauer stellt. So spricht beispielsweise Gerhard Ebeling ohne grösseres Aufheben von der Gebets- als einer "Ausnahmesituation" und vom Ge-

\footnotetext{
${ }^{14}$ Luhmann, Kommunikation mit Gott, 229.

${ }^{15}$ Ebd., 231.

${ }^{16}$ Ebd., 231f. Im letzten Satz des Zitats findet sich ein Druckfehler, den ich im Zitat von $>$ das $<$ nach $>$ daß< korrigiert habe.
} 
bet als einem "völlig einzigartige[n] Fall im Gebrauch der Sprache «" Man muss zwar hinzufügen, dass Ebeling den Kommunikationsbegriff vermeidet und das Gebet nicht über einen raumzeitlich lokalisierten, situativen Austausch konzipiert, sondern über eine Gott und Mensch verbindende Teilhabe an Sprachlichkeit. Aber suggeriert er nicht eben doch eine kommunikative Situation, wenn er seine Dialektik von Gottes Wort und menschlicher Antwort, von Angeredetsein durch Gott und menschlicher Anrede Gottes entfaltet? Macht er es sich - und wie oft: wir mit ihm - nicht auch ein bisschen bequem, wenn er einen vermeintlich einzigartigen Ausnahmefall in Anspruch nimmt und vor kritischen Nachfragen aus der offenen Lichtung des Regulären in das halbschattige Zwielicht eines sSo wie ... nur ganz anders ausweicht, wo er sagen und verstehen kann, was er will, ohne Rechenschaft geben zu müssen?

Nun ist es eines, aus einer distanzierten, normativen Perspektive heraus Kritik zu formulieren, und etwas anderes, eigene positive Aussagen zu treffen, die sich angesichts des Aspektreichtums gelebter Vollzüge bewähren müssen. Lässt sich Luhmann etwas tiefer auf theologische Fragestellungen ein und beschreibt konkrete Praktiken, so gerät er auf Denkwege, die in Spannung zu seinen normativen Intentionen stehen. Dies zeigt sich, wenn Luhmann erkundet, was an der Annahme einer Kommunikation mit Gott theologisch produktiv sein könnte. Er beobachtet: In der Kommunikation über Gott ist Raum für Leugnung und Streit. Und er fährt fort:

"In der Kommunikation mit Gott ist dagegen all diese Kontingenz ausgeschlossen: Man könnte und würde ja nicht mit ihm kommunizieren, wenn es ihn nicht gäbe. [...] Die Kommunikation mit Gott bescheinigt sich selbst die Existenz des anderen. [...] Kein Thema, keine Aussage über Gott könnte je diese Art von Gewißheit erreichen. ${ }^{18}$

Hier muss man Luhmann entschieden auf einen Widerspruch hinweisen. Oben war die Rede davon, dass die kontingenzabsorbierende Funktion der Kontingenzformel gefährdet wäre, würde man davon ausgehen, dass man mit Gott kommunizieren könne. Hier gesteht Luhmann nun aber ein, dass gerade der Vollzug einer Kommunikation mit Gott jene Funktion leistet, um die es ihm systemtheoretisch geht: um die Absorbierung von Kontingenz in Gestalt der Steigerung der Gewissheit, dass Gott wirklich ist.

${ }^{17}$ Gerhard Ebeling, Dogmatik des christlichen Glaubens I, Tübingen ${ }^{4} 2012,196$ und 201.

${ }^{18}$ Luhmann, Kommunikation mit Gott, 232. 
Die hier sichtbar werdende kontingenzabsorbierende Seite konkreter Gebetsvollzüge hätte Luhmann zur Einsicht verhelfen können, dass es vielleicht gar nicht so sehr die Gestalt des Gottesbildes ist, woran sich die funktionale Tauglichkeit der Kontingenzformel erweist, sondern das Mass an Lebendigkeit, mit der sie in den kommunikativen Vollzügen der Gesellschaft operational gegenwärtig ist - ein Zugang, welcher der aufklärerischen Verve einer abstrakten, systemtheoretisch fundierten Vernunftreligion gehörig den Stachel nimmt.

Wirft man noch einmal einen Blick in Ebelings Dogmatik, so wird augenfällig, welches Potential Luhmann an dieser Stelle verspielt. Ebeling schreibt:

"Die Sprache dient [...] hier [sc. im Gebet, d.V.] auch und vornehmlich dazu, dieses Gegenüber selbst als wirklich in Anspruch zu nehmen. Man kann nicht anders beweisen, daß man mit der Wirklichkeit Gottes rechnet, als indem man zu ihm betet. ${ }^{19}$

Für den hermeneutischen Theologen Ebeling ist diese Koppelung von Gottes Wirklichkeit und menschlichem Lebensvollzug eine Möglichkeit, in zeitgemässer und verantwortbarer Weise Aussagen über das Dasein Gottes zu machen und dabei allzu robuste objektivierend-metaphysische Modelle einerseits und allzu vage subjektivistische Konzepte andererseits zu meiden. Entsprechend nutzt Ebeling in seiner Dogmatik »das Phänomen des Gebets" als den "hermeneutischen Schlüssel der Gotteslehre ${ }^{20}$. Es wird also nicht eine metaphysisch grundierte, theoretisch-abstrakte Überzeugung als das theologisch Primäre und das Gebet als das aus dieser Überzeugung sekundär Folgende begriffen, sondern die lebendige Glaubensspraxis, $\mathrm{zu}$ der das Gebet wesentlich gehört, bildet die eigentliche Basis, auf der alle theologischen Aussagen über Gottes Sein aufruhen.

Kurz:Was Ebeling in seiner Entfaltung der Dogmatik als zentralen Dreh- und Angelpunkt nutzt, das fristet bei Luhmann das Dasein einer Randnotiz, die dort angesiedelt ist, wo seine eigene Theorie in die Nähe des eigenen Tellerrands gerät, ohne aber die Offenheit aufzubringen, mutig über diesen hinauszublicken.

Es ist überdies auffällig, dass er, sobald er über die kontingenzabsorbierende Funktion des Gebetes spricht, ganz selbstverständlich auch von einer 'Kommunikation mit Gott redet. Dabei gibt es so etwas seiner Ansicht nach doch überhaupt nicht! - Aber was ist dann ein tatsächlich vollzogenes Gebet? Ist es simulierte Kommunikation

\footnotetext{
${ }^{19}$ Ebeling, Dogmatik I, 202.

${ }^{20}$ Ebd., 193.
} 
oder Pseudokommunikation? Was unterschiede simulierte beziehungsweise Pseudokommunikation von echter Kommunikation? Wie kann sich eine Operation für Kommunikation halten, ohne Kommunikation zu sein? Und wie kann sich eine Operation sicher sein, Kommunikation zu sein und nicht simulierte beziehungsweise Pseudokommunikation?

Dass Luhmann es sich in dieser Sache recht leicht macht, bestätigt seine Vorlesung Einführung in die Systemtheorie. Dort trägt Luhmann vor:

"Kommunikation kommt überhaupt nur zustande, wenn jemand im Groben versteht oder vielleicht auch missversteht, aber jedenfalls so weit versteht, dass die Kommunikation weiterlaufen kann, und das liegt außerhalb dessen, was man durch die bloße Benutzung von Sprache schon sicherstellen könnte. Es muss jemand erreichbar sein, muss hören oder lesen können. ॥ $^{21}$

Es werden hier zwei Kriterien für das Zustandekommen von Kommunikation genannt. Das eine besagt, dass eine Instanz da sein müsse, die etwas versteht, gleichgültig, ob es ein Verstehen oder Missverstehen ist; das andere, dass Kommunikation fortgesetzt werden kann. Beide Kriterien setzen die Perspektive eines allwissenden Beobachters voraus. Innerhalb des Systems kann eine involvierte Instanz jedoch in manchen Fällen nicht sicher sein, ob die Kommunikation wirklich abgebrochen oder ob sie nur noch nicht weitergegangen oder anderswo als intendiert weitergegangen ist. Deshalb müsste Luhmann irgendwie berücksichtigen, dass es Phänomene gibt, welche aus Sicht des Systems durchaus eine Art Kommunikation sind. Sie sind es deshalb, weil Kommunikation an sie anschliessen kann. Ich denke an Phänomene wie das angeredete liebe Tagebuch, der nicht verschickte Brief, die an einen Verstorbenen adressierte Abdankungsrede, das Lied für die ferne Geliebte oder der an den Weihnachtsmann verfasste Wunschzettel. Es gibt ja Instanzen, die möglicherweise verstehen: derjenige, der das Tagebuch verbotenerweise liest, diejenige, die den Brief zufällig findet, die Hörerinnen und Hörer der Abdankungsrede oder die Eltern des wunschzettelschreibenden Kindes. Und wenn dann das Gewünschte unter dem Baum liegt, so ist es für das Kind Kommunikation mit dem Weihnachtsmann und für die Eltern uneingestandene Kommunikation mit dem Kind - und Luhmann müsste beides abbilden können, weil das Kind mit seinen Freunden darüber in einer und die Eltern mit

${ }^{21}$ Niklas Luhmann, Einführung in die Systemtheorie, hg. v. Dirk Baecker, Heidelberg ${ }^{6} 2011,76 f$. 
den Grosseltern in anderer Weise weiterkommunizieren werden. Und wenn dann die Eltern mit dem Kind über den Weihnachtsabend sprechen, so findet eine Kommunikation statt, auf der es viele und in Spannung zu einander stehende Ebenen des Verstehens und Nichtverstehens gibt.

Meine Überlegungen wollen hier lediglich deutlich machen, dass Luhmann zur Frage, ob ein Gebet Kommunikation ist, doch erheblich mehr sagen müsste. Es findet im Religionssystem ja einiges an Kommunikation über diese Frage statt, und vor dem entsprechenden Hintergrund muss man auch ein konkret praktiziertes Gebet sehen. Pointiert gesagt: Das Gebet kennt den Zweifel und kann in sich selbst die Unsicherheit darüber, ob Kommunikation stattfindet, ja durchaus abbilden.

\section{Zwei Kommunikationsmodelle}

Diese Ausführungen legen es jedenfalls nahe, dass der Kommunikationsbegriff im besprochenen Text nicht ganz so klar ist, wie durch Luhmann suggeriert wird. Interessanterweise kommt er selbst am Ende seines Textes noch einmal auf die Frage nach einem angemessen Verständnis von Kommunikation zurück. Er schlüpft wiederum in die Rolle des gönnerhaften Freundes der Theologie und fragt:

»Wird die Religion gegen alle Plausibilität an der Möglichkeit einer die Gesellschaftsgrenzen transzendierenden Kommunikation festhalten (was durchaus möglich ist, aber Lasten mit sich bringt) oder nicht? / Wenn man diese Frage ernst nimmt, wird es um so wichtiger, genau zu sagen, was man unter Kommunikation verstehen will, und auch in dieser Hinsicht könnte die Soziologie zu denken geben. $\mathbb{1}^{22}$

Was die Soziologie der Theologie zu denken zu geben hat, wird wenig später klar: nichts weniger als Niklas Luhmann und seine Kommunikationstheorie. Diese zeichnet sich dadurch aus, dass Kommunikation abstrakter gefasst und von einem 'Zwei-Personen-` $\mathrm{zu}$ einem ’Drei-Selektionen-Modell übergegangen werden soll. Dieses wird in folgenden Worten zusammengefasst:

"Von Kommunikation kann immer dann gesprochen werden, wenn drei Selektionen, nämlich eine Information, eine Mitteilung und das Verstehen zusammentreffen und sich wechselseitig

${ }^{22}$ Luhmann, Kommunikation mit Gott, 233. 
bestätigen. Kommunikation ist demnach eine Synthese aus drei Selektionen, ist eine zunächst recht unwahrscheinliche, emergente Einheit, die aus hochkontingenten Einzelereignissen gebildet wird. [...] Jede Einzelkommunikation bleibt als isoliertes Ereignis extrem unwahrscheinlich. Nur der Zusammenhang vieler Kommunikationen und die dabei entstehenden Erwartungsstrukturen transformieren diese Unwahrscheinlichkeit in Wahrscheinlichkeit. Jede Kommunikation dient dann zugleich als Bedingung der Möglichkeit anderer Kommunikationen. So entsteht ein rekursiver, selbstreferentieller Zusammenhang, und eben das ist Gesellschaft. «23

Es gibt bessere Zusammenfassungen ${ }^{24}$ und öffnend-hilfreiche Interpretationen $^{25}$ von Luhmanns Modell, auf die ich nun eingehen könnte. Im hier gesetzten Rahmen muss die Erläuterung genügen, dass Kommunikation nicht mehr als isolierter Übermittlungsvorgang begriffen wird, in dem ein Subjekt A an ein Subjekt B eine Nachricht übermittelt. Dies entspräche dem von Luhmann kritisierten klassischen Zwei-Personen-Modell. Kommunikation wird dem gegenüber verstanden als ein komplexes Operieren, das Differenzen in Anspruch nimmt bzw. Differenzen generiert: ein Operieren, das Auswahlen aus Auswahlbereichen trifft bzw. eine Unterschiedenheit von Auswahlen, die getroffen werden, und Auswahlbereichen, aus denen ausgewählt wird, erzeugt. Dieses Operieren hat mit Information, Mitteilung und Verstehen drei Aspekte, wobei die Pointe darin liegt, dass eine Information nur Information sein kann vor dem Hintergrund anderer möglicher Informationen, eine Mitteilung (im Sinne des Trägers der Information) vor dem Hintergrund anderer möglicher Mitteilungen und ein Verstehen vor dem Hintergrund anderer möglicher Verständnisse. Damit wird auch deutlich, warum mit einem Kommunikationsvollzug stets das Ganze der Gesellschaft thematisch wird: weil sich Kommunikation nur ereignen kann vor dem Hintergrund all dessen, was in einer Gesellschaft und ihren Teilsystemen an möglicher Kommunikation erwartet wird.

Es fällt auf, dass in Luhmanns Auseinandersetzung mit dem Gebet praktisch nichts von seinem eigenen kommunikationstheoretischen Ansatz eingeflossen ist. Genauer gesagt: Luhmann nutzt das Gebet nicht, um die Leistungsfähigkeit des von ihm entwickelten Drei-Selektionen-Modells unter Beweis zu stellen und etwa das Gebet anders zu interpretieren als er glaubt, dass die Theologen es

${ }^{23}$ Luhmann, Kommunikation mit Gott, 233.

${ }^{24}$ Vgl. Luhmann, Einführung in die Systemtheorie, 277-302.

${ }^{25}$ Vgl. Dirk Baecker, Form und Formen der Kommunikation, Frankfurt a.M. ${ }^{3} 2013$. 
interpretieren würden. Das Gebet fungiert bei ihm viel eher als Exempel, um die Mangelhaftigkeit des von ihm so scharf kritisierten Zwei-Personen-Modells zu thematisieren. Entsprechend äussert er am Ende seines Textes mit Stirnrunzeln, dass »die christliche Religion sehr komplexe Verhältnisse zu einem `Zwei-Personen`-Modell kondensiert $\ll^{26}$ habe - womit implizit gesagt ist, dass die Theologie das in seinen Augen hochproblematische Modell in Gestalt der Gott-Mensch-Relation gewissermassen zu einem die Statik der christlichen Religion tragenden Element gemacht habe und nun hilflos daran herumwerkele. Entsprechend endet der Text damit, dass die Theologie vor die Wahl gestellt wird, entweder die »Distanz zwischen Mensch und Gott immer weiter zu vergrößern" oder "die Vorstellung einer kommunikativ erreichbaren Transzendenz ganz auf[zu]geben $\aleph^{27}$.

Um es auf den Punkt zu bringen: Fragt Luhmann, ob das christliche Gebet als Kommunikation mit Gott gedacht werden könne, so spricht er von Kommunikation im Sinn des von ihm kritisierten Zwei-Personen-Modells. Spricht er über seine eigene Religionstheorie etwa hinsichtlich der Verwendung der Kontingenzformel Gott, so steht sein eigenes Drei-Selektionen-Modell im Hintergrund. Das ist problematisch, und die von Luhmann angemahnte Klärung des Kommunikationsbegriffes hätte der Konsistenz seiner eigenen Überlegungen gut getan. Denn dann wäre er vielleicht selbst auf diese Fragen gestossen: Ist der Gedanke einer Kommunikation mit Gott auch dann unzulässig, wenn man anstelle des Zwei-Personendas Drei-Selektionen-Modell zu Grunde legt? Kann man das, was Ebeling in seiner Dogmatik entfaltet, ohne Weiteres auf das ZweiPersonen-Modell beziehen? Kritisiert Ebeling nicht auf seine Weise das Zwei-Personen-Modell? Und hängen Luhmanns Überlegungen zum Gebet nicht völlig in der Luft, so lange der kommunikationstheoretische Status der nach seiner Ansicht illegitimen, aber gleichwohl praktisch vollzogenen Gebetspraxis nicht mit Bezugnahme auf das Drei-Selektionen-Modell geklärt ist?

\section{Ausblick und Fazit}

Bis hierhin stand die kritische Auseinandersetzung mit Luhmann im Fokus. Zum Abschluss soll nun ein konstruktiver Vorschlag unterbreitet werden, in welcher Richtung man weiterdenken könnte,

\footnotetext{
${ }^{26}$ Luhmann, Kommunikation mit Gott, 234.

27 Ebd., 235.
} 
um die Stärken des Drei-Selektionen-Modell für eine Deutung des christlichen Gebets auszunutzen, ohne Luhmanns starke, normativen Intentionen geschuldete Abwertung zu übernehmen.

Zunächst müsste man natürlich versuchen, das Gebet nicht als eine Gesprächssituation zwischen zwei Subjekten, einem menschlichen und einem göttlichen, zu denken. Es müsste vielmehr darum gehen, es als einen Kommunikationsvollzug zu begreifen, der sich im religiösen Subsystem der Gesellschaft vollzieht und dabei vorhergehende Kommunikation aufgreift, parallele Kommunikation begleitet und weitere Kommunikation nach sich zieht. Das bedeutet, dass der Sinn des Gebets ganz aus dem gemeinschaftlich geteilten Vollzug des christlichen Lebens heraus verstanden werden müsste und nicht aus einer solitären - möglicherweise mit einem problematischen metaphysischen Verständnis einhergehenden - Konstellation von immanentem Mensch-Subjekt und transzendentem Gott-Subjekt.

Weiter müsste entfaltet werden, inwiefern sich das Gebet in Form und Inhalt von andern Kommunikationsoptionen christlichen Lebens unterscheidet. Das heißt: Wer sich dafür entscheidet, zu beten, der entscheidet sich zugleich dagegen, ein Bekenntnis abzulegen, die Bibel zu lesen, sich in einem Hauskreis über den Glauben auszutauschen, eine Dogmatik zu schreiben, ein Seelsorgegespräch zu suchen, eine Predigt zu hören, die Eucharistie zu feiern, im Kloster $\mathrm{zu}$ meditieren, diakonisch tätig zu sein und so weiter und so fort. Was ein Gebet auszeichnet, das zeichnet es nur in Abgrenzung von und in Beziehung auf all die anderen Kommunikationsgestalten des christlichen Glaubens aus - und dies zeigt sich in besonderer Weise darin, dass manche dieser Kommunikationsoptionen dem Gebet überaus nah stehen, aus ihm heraus oder in es hineinführen oder es sogar in sich integrieren bzw. integrieren können.

Inhaltlich vollzieht sich im Gebet - wie auch in anderen Kommunikationsgestalten des christlichen Glaubens - eine (Selbst)Beobachtung des Lebens im christlichen Glauben. Das bedeutet, dass die Unterscheidungen, welche in der Kommunikationsgemeinschaft der Glaubenden operational präsent sind, auf ein konkretes Leben angewandt werden, also dieses Leben etwa hinsichtlich der Fragen Sünde und Vergebung, Glaube und Anfechtung, Tod und Auferstehung und so weiter beobachtet und in den entsprechenden Spannungsverhältnissen lokalisiert wird.

Die eigentliche Pointe des Gebets liegt allerdings im Formalen: dass sich diese Selbstbeobachtung des Lebens im christlichen Glauben als eine vollzieht, die an Gott adressiert ist. Dabei stemmt sich 
die Anrede gegen die Kontingenz des Gottesgedankens und gibt der geglaubten Wirklichkeit Gottes eine lebendige Gestalt im Medium der Sprache. Um mit Luhmann zu sprechen: Die Kontingenzformel Gott wird zwar als transzendent geglaubt, doch vollzieht sich dieses Glauben in immanenten Kommunikationsakten. Das Gebet leistet also die von Luhmann beschriebene kontingenzabsorbierende Vereinheitlichung von Immanenz und Transzendenz in besonderer Weise und spielt gerade deshalb in religiösen Vollzügen zu Recht eine herausragende Rolle. Man könnte sich daher beinahe fragen, ob nicht Gott in Luhmanns Theorie als Kontingenzformel fungieren sollte, sondern der angebetete Gott.

Dabei ist wohlgemerkt auch die transzendente Entzogenheit des angebeteten Gottes im immanent sich vollziehenden Kommunikationsakt des Gebets präsent. Dies wird besonders anschaulich in den metakommunikativen Elementen des Gebets, ${ }^{28}$ also in solchen, in denen sich die Kommunikation selbst als Kommunikation thematisiert. In solchen Elementen kommuniziert das Gebet über sich selbst in seiner eigenen Unselbstverständlichkeit. Die beiden prägnantesten sind, dem Anredecharakter Rechnung tragend, als Bitten formuliert: >Lehre mich beten! (vgl. Lk 11,1) und `Erhöre dieses Gebet! (zum Beispiel Ps 4,2f). Das bedeutet, dass die Basis des Gebets, also die Frage, ob und wie legitim zu beten ist, und die Konsequenz des Gebets, also die Frage, was das Gebet im Leben im Glauben nach sich zieht, nicht von der Immanenz her gesichert werden kann, sondern der gnadenhaften Initiative Gottes anheim gestellt ist.

Ich lasse es mit diesen Andeutungen bewenden, um noch kurz $\mathrm{zu}$ meiner einleitenden Frage nach den Möglichkeiten eines produktiven theologischen Umgangs mit Luhmann zurückzukehren. Es dürften in den zurückliegenden Erörterungen sowohl die Grenzen als auch die Chancen einer Luhmann-Rezeption in der Theologie deutlich geworden sein. Ich ziehe aus den obigen Überlegungen den Schluss, dass Luhmann seine eigene Intention, nämlich die Religion als autopoietisches System kommunikativen Operationalisierens zu beschreiben, nicht konsequent genug verfolgt. Bisweilen vermischen sich deskriptive und normative Perspektiven allzu stark, und mitunter wird ein Bild von christlicher Religion gezeichnet, das

\footnotetext{
${ }^{28}$ Es wird auch etwa in der Hioberzählung deutlich. In seiner klagenden Anrede Gottes versucht Hiob, Gott in die Kontingenz zu ziehen und mit ihm über das, was geschehen ist, zu diskutieren. Doch die Kontingenzformel beharrt auf ihrer kontingenzabsorbierenden Entzogenheit, indem sie aus einem Wettersturm heraus die eigene Position unmissverständlich klarstellt.
} 
nicht dem Stand gegenwärtigen Theologisierens und der faktischen Komplexität der Phänomene entspricht.

Insbesondere dann, wenn Luhmann über den dualen Code von Immanenz und Transzendenz spricht, neigt er dazu, in einen metaphysischen Ansatz zurückzufallen, den er doch selbst hinter sich lassen will, wenn er beispielsweise schreibt: "Alles, was es gibt, beruht von einem operationalen Theorieansatz her gesehen auf demselben Grundvorgang, demselben Typ von Ereignis, nämlich auf Kommunikation. ${ }^{29}$ Entsprechend wäre es für die Theologie produktiv, Luhmann so weiterzuinterpretieren, dass auch die immanente Thematisierung des transzendenten Jenseits nicht ein metaphysisches Jenseits kommunikativ zu erreichen trachtet, sondern selbst ein Ereignis der kommunikativ operationalisierenden Immanenz ist. Auch die Existenz der Transzendenz müsste von Luhmann stärker als ein Ereignis der Kommunikation behandelt werden, das heisst ohne noch einmal die Möglichkeit eines metaphysisch existierenden Jenseits ins Spiel zu bringen, wie er es im hier behandelten Text getan hat.

Meine Überlegungen haben darüber hinaus zumindest exemplarisch gezeigt, dass durchaus Spielräume für produktive und kritische Luhmann-Rezeptionen in der Theologie bestehen. Man muss sich von der Grösse und Engmaschigkeit des systemtheoretischen Gedankennetzes also nicht allzu sehr beeindrucken lassen. Schon allein die Einsicht in das nicht immer klare Verhältnis deskriptiver und normativer Aspekte reisst gehörige Lücken in Luhmanns Flechtwerk, was diverse Ansatzpunkte für kritisch-korrigierende und in der Folge eigene freie Überlegungen bietet.

Neben solchem eher kleinteiligen Auftrennen und Anknüpfen gibt es eine weiträumiger denkende Alternative. Man kann versuchen, die sich breit ausspinnende Gedankenwelt Luhmanns auf die Logik ihrer Maschenordnung hin zu befragen, um dann mit Hilfe des so abgeschauten Wissens über Luhmannknoten ein eigenes, neues Netz zu knüpfen. Ein solches könnte die Möglichkeit bieten, gewisse Theorieelemente zu abstrahieren und gewissermassen von Luhmanns positiven und negativen Vorurteilen über die Religion zu lösen, um diese Elemente dann noch einmal neu und geleitet von eigenen Intentionen auf religiöse Phänomene anzuwenden. Dafür kämen etwa die entsubjektivierte Kommunikationstheorie oder der Spencer-Brown weiterinterpretierende Komplex in Frage, der sich zwischen Beobachtung und Reentry aufspannt. Und möglicherweise könnte man sich so in Anlehnung an Luhmanns Gedanken und

${ }^{29}$ Luhmann, Einführung in die Systemtheorie, 76. 
zugleich in eigenständiger und theologisch selbstbewusster Weise auf die offene See hinaus wagen, um auf Fang zu gehen.

- Dr. Stefan Berg ist wissenschaftlicher Mitarbeiter am Institut für Hermeneutik und Religionsphilosophie der Universität Zürich. Er arbeitet an einem Habilitationsprojekt zur Erwählungslehre und theologischen Unterscheidungsoperationen, finanziert durch den Forschungskredit der Universität Zürich und den Schweizerischen Nationalfonds. 